\title{
Energy Exchange Between Laser Pulses in Atomic Medium with a Closed Excitation Contour
}

\author{
K.A. Barantsev, A.N. Litvinov, and E.N. Popov ${ }^{a}$ \\ Peter the Great St. Petersburg Polytechnic University, 29 Politechnicheskaya, St. Petersburg 195251, Russia
}

\begin{abstract}
In this work we investigate propagation of two laser pulses in the optically dense atomic medium (three-level $\Lambda$-system) in the case of a closed excitation contour. The closed excitation contour is created by additional microwave radiation which interacts with the lowfrequency transition of the $\Lambda$-system. Two cases are considered: when the pulse duration is more and less then lifetime of the excited atomic level. It is shown that the propagation of the pulses depends on the relative phase of the fields. When the relative phase is zero, the pulses energy is absorbed linearly. When the relative phase is $\pi / 2$, the pulses exchange energy between each other.
\end{abstract}

Keywords: closed excitation contour, laser pulse, coherent population trapping, optically dense medium

In the last two decades a lot of works has been devoted to the coherent population trapping effect (CPT) [1]. The essence of this effect is the appearance of a specific superposition of long-lived states in a three-level atom ( $\Lambda$-system) which is excited by two-frequency radiation. This superposition does not interact with radiation. It leads to disappearance of the absorption in a certain frequency band (area of the CPT-resonance).

When the CPT-resonance is excited by two-frequency electromagnetic field, the propagation of continuous radiation obeys a linear law [1]. At the same time the CPTresonance becomes sensitive to the relative phase $\Phi=\varphi_{1}-\varphi_{2}-\varphi_{3}$ of the fields when the third field takes place due to appearance of the closed excitation contour. It is known that in the case of $\Phi=0$ the CPT-resonance takes place and when $\Phi=\pi / 2$ it collapses [2]. In the work [3] for continuous radiation it has been shown that the total intensity decreases according to exponential law for $\Phi=\pi / 2$, but the frequency components of the field exchange energy between each other.

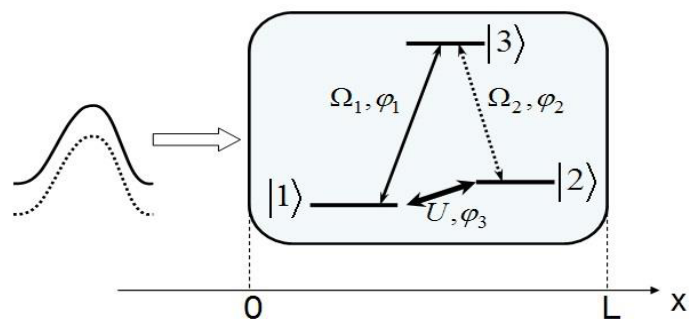

Figure 1. Medium of three-level atoms which interacts with continuous electromagnetic field $U$ and two pulses $\Omega_{1}$ and $\Omega_{2}$ with the initial phases $\varphi_{1}, \varphi_{2}$ and $\varphi_{3}$. The fields form a closed excitation contour.

${ }^{a}$ Corresponding author: kostmann@yandex.ru 
It leads to oscillating law of propagation for each of the frequency components.

In the present work absorption of the pulsed radiation is analyzed when a closed excitation contour takes place. Let us consider the medium of motionless three-level $\Lambda$-atoms (Fig.1) which interact with two synchronous laser pulses with Rabi frequencies $\Omega_{1}$ and $\Omega_{2}$ which are resonantly tuned to the transitions 1-3 and 2-3. The transition 1-2 is bound by microwave field $U$. Propagation of the laser pulses through the medium is described by the Maxwell-Bloch equations for slow amplitudes of the electric field $E_{j}^{0}(x, t)$ and atomic density matrix $\rho_{m n}(x, t)$ :

$$
\left\{\begin{array}{c}
\frac{\partial E_{j}^{0}(x, t)}{\partial x}+\frac{1}{c} \frac{\partial E_{j}^{0}(x, t)}{\partial t}=4 \pi P_{j}^{0}(x, t) k_{j} \\
\frac{\partial \rho_{i k}(x, t)}{\partial t}=-\frac{i}{\hbar} \sum_{l}\left[H_{i l} \rho_{l k}(x, t)-\rho_{i l}(x, t) H_{l k}\right]+\sum_{l, m} \Gamma_{i k, l m} \rho_{l m}(x, t)
\end{array},\right.
$$

where $k_{j}$ are the wave numbers, $H$ is the Hamiltonian, $\Gamma$ is the relaxation matrix, $j=1,2 . P_{j}^{0}(x, t)$ is the slow amplitude of polarization of the medium. The microwave field $U$ does not decay in the medium due to small electric dipole moment.

On the Fig.2 the dependence of normalized pulse energy $W_{i}(x)=\int_{-\infty}^{\infty}\left|\Omega_{i}(x, t)\right|^{2} d t / \int_{-\infty}^{\infty}\left|\Omega_{i}(0, t)\right|^{2} d t$ on coordinate $x$ is shown.
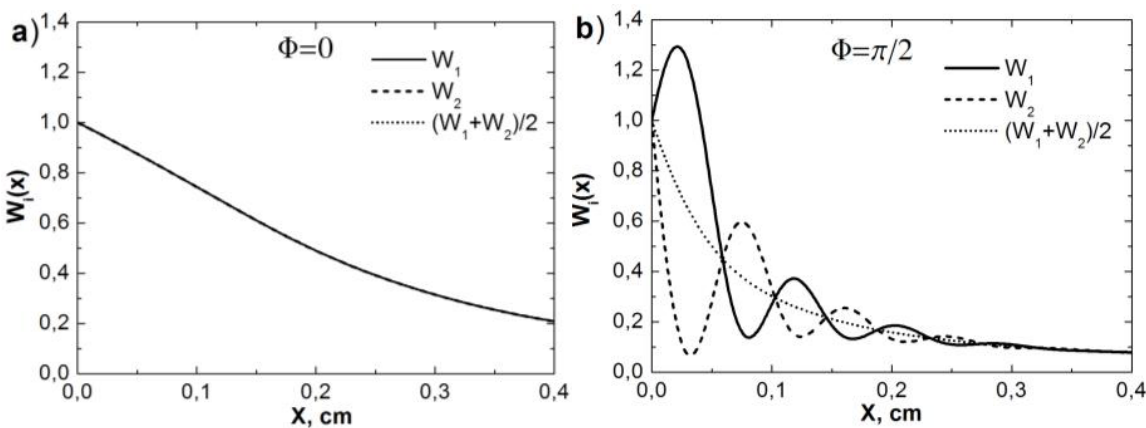

Figure2. Dependence of normalized energy of the $i$-th pulse on coordinate $x$ for relative phase $\Phi=0$ (a) and $\Phi=\pi / 2$ (b) for the Gaussian pulses. Duration of the pulses is $4 \gamma^{-1}$ and amplitude is $2 \gamma$, where $\gamma$ is the rate of spontaneous relaxation of the excited level.

One can see that synchronous absorption of the pulses takes place when the relative phase is zero $(\Phi=0)$. Energy of the pulses decreases linearly (Fig.2(a)). In the case of $\Phi=\pi / 2$ the pulses exchange energy between each other (Fig.2(b), solid and dashed curves). At the same time the total energy decreases exponentially (dotted curve). This effect is in agreement with the results for continuous radiation [3] while striving pulse duration to infinity.

This work was supported by State Assignment No. 2014/184, State Assignment No. 3.1446.2014K, Russian Foundation for Basic Research (projects № 14-02-31422, № 15-02-01013, № 13-02-00944).

\section{References}

1. B.D. Agapiev, M.B. Gorniy, B.G. Matisov et al., UFN. 163, No 1 (1993)

2. L. Hebin, V.A . Sautenkov, Yu.V. Rostovtsev et al., Phys. Rev. A. 80, 023820 (2009)

3. K.A. Barantsev, A.N. Litvinov, JETP. 118, Iss. 4., p.569-579 (2014) 